\title{
PROTOTYPE FOR A MESSAGE: AMBULATORY CONSULTATION BASED OD A HL7 STANDARD FOR TELECONSULTATION
}

\author{
LILIA E APARICIO P, JAIME A BENÍTEZ \& MIGUEL A ÁVILA A \\ Engineering Faculty, Universidad Distrital Francisco José de Caldas, Bogotá, Colombia
}

\begin{abstract}
Within the framework of the research for development of telemedicine at the global level, the standard HL7, that corresponds to one of the lines of global integration of the necessary infrastructure for the supply of services in telemedicine, is an environment of integration and interoperability of an information system that along of the technological development, have yielded results that today are in production in the different Colombian institutions in multiple information systems not related. Particularly in this work, the methodology of construction of messages outpatient consultation, which will be integrated to the system of standardization in the case of tele-consultation applied to the case of Colombia, is part of the macro projected HL7, tele-consultation and tele-diagnosis in Colombia accordance with existing regulations and legalization of consultation services and diagnosis, that they are already regulated in the field of health in Colombia. In the HL7 standard, it is necessary to design and develop the messages from each of the different scenarios for the exchange of electronic information, as it is consultation ambulatory with the aim of improving efficiency in the delivery of information to the end user.
\end{abstract}

KEYWORDS: HL7, Telemedicine, Messages, ambulatory consultation

Received: Jun 08, 2020; Accepted: Jun 28, 2020; Published: Oct 10, 2020; Paper Id.: IJMPERDJUN20201500

\section{INTRODUCTION}

On the frame of the research for the development of the telemedicine on a global scale, the HL7 standards that correspond to each one of the guidelines of global integration of the needed structure to the offer of services of telemedicine, one an environment of integration and interoperability of the information system, that throughout the technological develop have given results that today are seen on productions of the different Colombian institutions, on multiple systems of no integral information.

There is the international organization HL7, which is responsible of create and support the standards of the health sector, which allows an integration of different entities systems that provide the health service to have a better attention to the user and have a better effectiveness on the development of their need process for the health service.

On the Colombian case, it is necessary to develop the Messenger of each one of the components of the system of health management, where each one of them has a considerable complexity, that why it is necessary to plan a macro Project that allows the integration of various Works, where each of them correspond to a subgroup of messages, to its development it is necessary to appropriate the reference and the definition of the identifiers of the 
standard adapted to Colombia.

On this particular work, it is going to be develop the methodology of construction of the message of ambulatory consultation which is going to be integrated to the standardization system for the case of teleconsultation applied to the Colombian case, which is part of the macro Project HL7 of teleconsultation and telediagnostic on Colombia according to the actual normativity and the legalization of the consultation and diagnostic services, that are already regulated on the ambit of health in Colombia, regulated by organization such as the minister of social protection and the implementation of services of telemedicine in Colombia.

In the HL7 standard, is necessary to design and develop the messages of each one of the different sceneries for the Exchange of electronic information, as the ambulatory consultation with the objective of making better the delivery of information to the final user.

\section{RESEARCH METHODOLOGY}

For the development of this research, it must be considered the following methodologies:

The first one correspond to the development of the message on a good quality atmosphere for the software and the second correspond to the methodology proper of analysis for the messenger HL7.

Phase 1: Preliminary investigation: On this phase, it will be made a standard study, where it will be analyzed other applications (in other countries), the regulation and the Colombian legislation.

Phase 2: Analysis and specification: On this phase, it will be made a detailed exploration of the standard and for the parts that compound the standard.

Phase 3: Design and construction of the message: On this phase, it will be working directly with the scheme of the message HL7, according with the design to the Colombian case, and according to the descriptions of the ballot and the CDA.

Phase 4: Evaluation: On this phase, it will be running the proof for the validation of the message.

Phase 5: Modification: According to the results of the proof.

Phase 6: Technical design: It will be deliver the implementation guide.

Phase 7: Programming and proof: It integrated the message to the global system of teleconsultation and telediagnostic.

On respect to the methodology of the HL7 message, it must be developed the following stages (parallel to the phases of development of the prototype):

Phase 1: Definition of the specifications: On this first phase, the objective is to audit the information relative to the problem, with the purpose of collet the necessary data for the resolution.

Phase 2: Conceptual design: The objective of this phase is to construct a model of information that reflects the conceptual schedule of the prototype.

Phase 3: Prototype development: This phase is made to construct the first prototype of the operative for the application 
Phase 4: Proves for the user: On this phase, it will be made all the necessary proves to validate the prototype develop on phase 3

Phase 5: Implementation: On this phase, the plan of formation of the users will be executed, and it will be carried out the process of migration of the real execution of the application. After the finalization of the migration, it will be made the final proves, as activities to make final correction, checking out all the documentation for the Project.

\section{STRUCTURE FOR THE RESEARCH WORK}

The development of this degree work is carried out according to three specific objectives, raised to comply the general objective. Equally, the construction of the reference framework was realized based on the investigation research done as well as the investigation records.
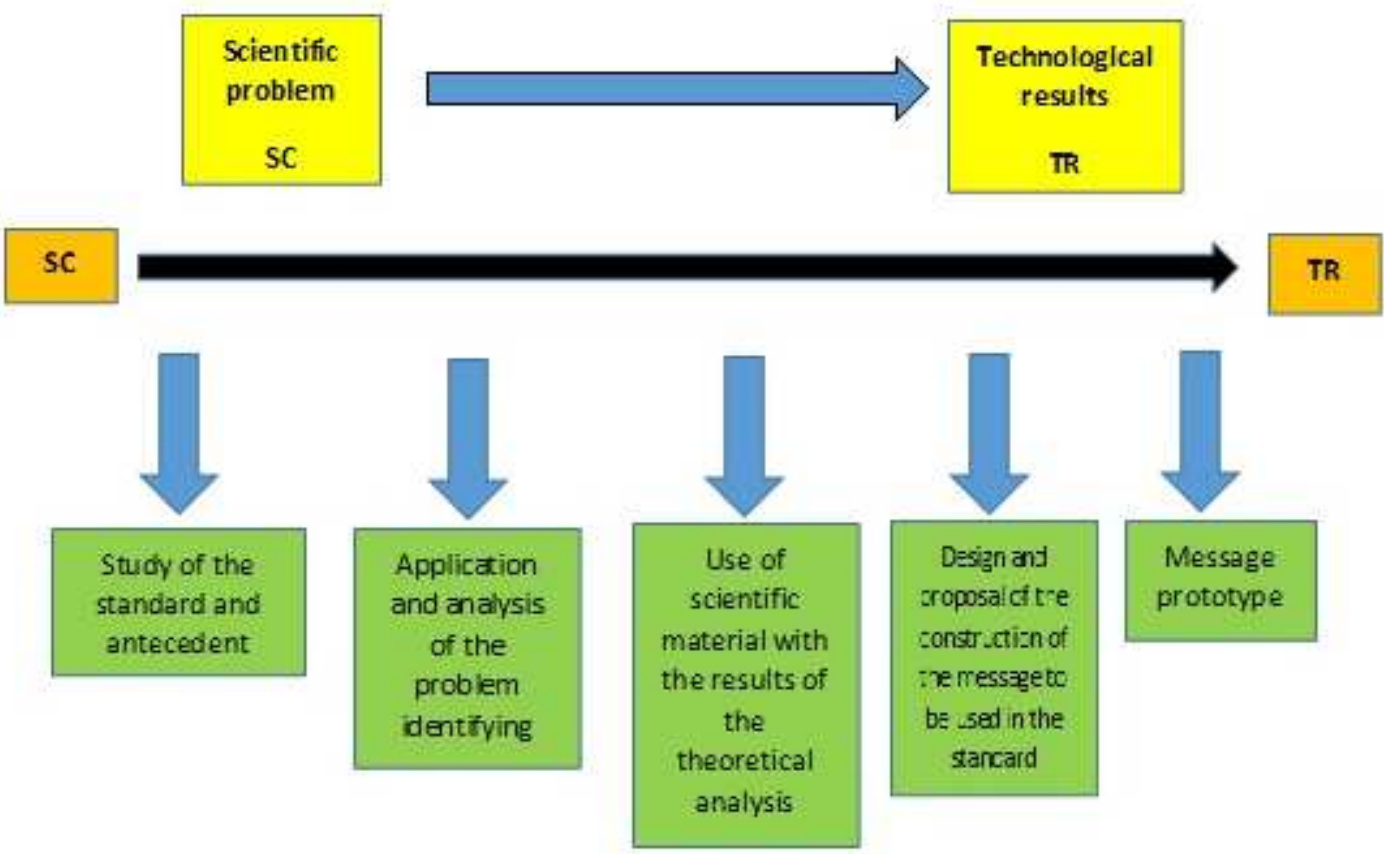

Figure 1: Structure of the research work. Source: Authors, 2011

The structure of the research work, was made starting off a scientific problem for reaching technological results, these types of results should study the state of the standard, to the case HL7, once the problem its identify it must arrive to an appropriation and analysis of the problem by using scientific material to get to a prototype of message to the real world, the prototype of message is an ambulatory consultation.

All the institutions that provides the health service need different systems to their internal control, like billing, medical agenda, radiology and so on. Each one of these systems need the information of other systems to function in the right way, on this moment each system works in an independent way making it slower on the prosecution of information to the different process need to the good attendance of the patient.

In order to the systems of the health entities to be more efficient there is need an operability between them, in that moment the HL7 its implement, because it allows us to integrate systems that uses different semantics, and led us to make them interact, sharing electronical information to improve the effectiveness of the processes, besides it allows to share trustworthy information through the red, where each doctor with the necessary knowledge has the capability to analyzed 
and take benefit of this information.

It's an initiative that start on 1987, based on interface problems that get presented between the multiple information systems, over time it become on a standard for the electronic exchange of clinical data and administrative information on the health campus of the United States [1] .

The international organization of standards, stablish that to be able to exist any Exchange of information its necessary to exist some syntactic and semantic rules previously agreed, where is made capture information, storage, and transmission of information that is indispensable to define the set of rules, that allows to the exchange of information of the people with the final purpose of improve the of the health care.

On the following decades, the health institutions were automated their process with the final proposed of get lower consumption of paper and to be more efficient in the moment of given required information for the patients, initially systems of information were created about the consultation of patients, later the administrative part, these systems had an independent functioning that was why it could not ley the share of information between them.

The HL7 standard represents a breakthrough in the implementation of system interoperability solutions in the healthcare environment. In version 2.x, it provides the data model of the messages that are exchanged by the different information systems as well as the integration events associated with them [2]

The today world is gifted by a large variety of standards to let them communicate between them, the HL7 is one of them and allows the interoperability between different information medical systems it guarantee that the information that is shared is more trustworthy.

The objective of the HL7 is to be allowed the Exchange of data that generates the process of medical information through the development of guides, methodologies and general services offering interoperability between systems of health information in a flexible and efficient way for the management of information.

This is accomplished because it allowed the Exchange of information between applications developed for the different providers of software, reduce the paper work, gets better the support of decisions and allows the integration of health information through the time, between different services. It allows the connectivity between heterogeneous systems with competitive costs, offering flexibility, because it can be implemented using diverse types of software technology. It reduces the inverted resources on the negotiation of the interfaces between applications, it reduce the invert resources of programming and the maintenance of proprietary interfaces. 


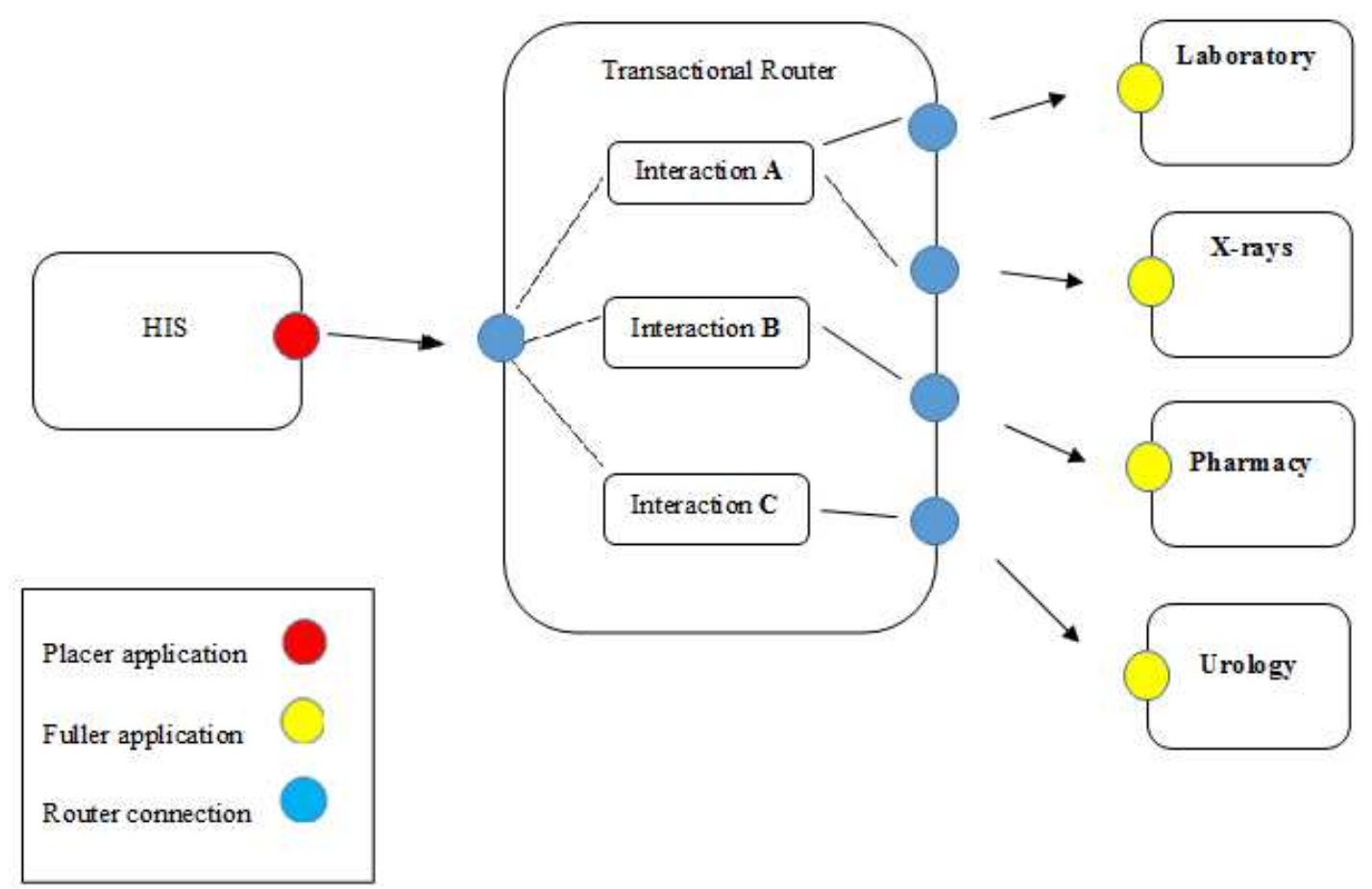

Figure 2: Structure of the HL7 [3].

The HL7 started on 1997, like a fundamental stake out of the standard of health under an HL7 volume 3, developing one model of referential information (RIM), this is the base of the Exchange of information of the new standard, a system of dominium of the health version 3 that stimulates the terminologies registered and approved to be used with more frequency on the information systems. [3].

On this new version, the message structure it's derivate strictly of the information structure express on the RIM, certain types of predefined data, that jointly face the semantics to describe defined or undefined, complete or partially complete amounts codified.

The evolution of the HL7 standards are made through chapters, it counts with specialized personal that conformed the committees, there is establish different methodologies where the members made its contribution to defined the draft versions, the versions are approved using the voting process HL7 (BALLOT).

When an approbation is made, it is combined like a prelaminar standard, this result its carried to another voting process, this voting its done by the web, the only condition it's to be but of the HL7 and have an advance knowledge of the topic. The prelaminar standard is positively approved, there is made final proves and then it is published.

All around the world they are various initiatives that are being develop about information standard son the health area. On these initiatives, the HL7 highlights because:

It is widely tested; the HL7 is already implemented don some parts of the Word such as Canada, United States, Japan, Australia, and some parts of Europe. Recently it has been created the HL7 affiliated in Brazil where it was carried implementation projects.

It is versatile; it gives answers to the needs of the different types of health services, independently of the level (hospital, municipality, province, etc.) or area (patient administration, laboratory, pharmacy, etc.). 
It is open, the HL7 it is a standard no owner developed by users, software providers and the rest of interested on the information system for the health area.

It is recognized, the HL7 has formal alliances with other organizations that develop standard and are represented on the initiatives of international standards like the ISO (Technical Committee for Health Information of the International Organization for Standardization)

On a cooperative atmosphere, where all the actors of the system can participate, the HL7 can cover the changing necessities that the actual health information system has [4].

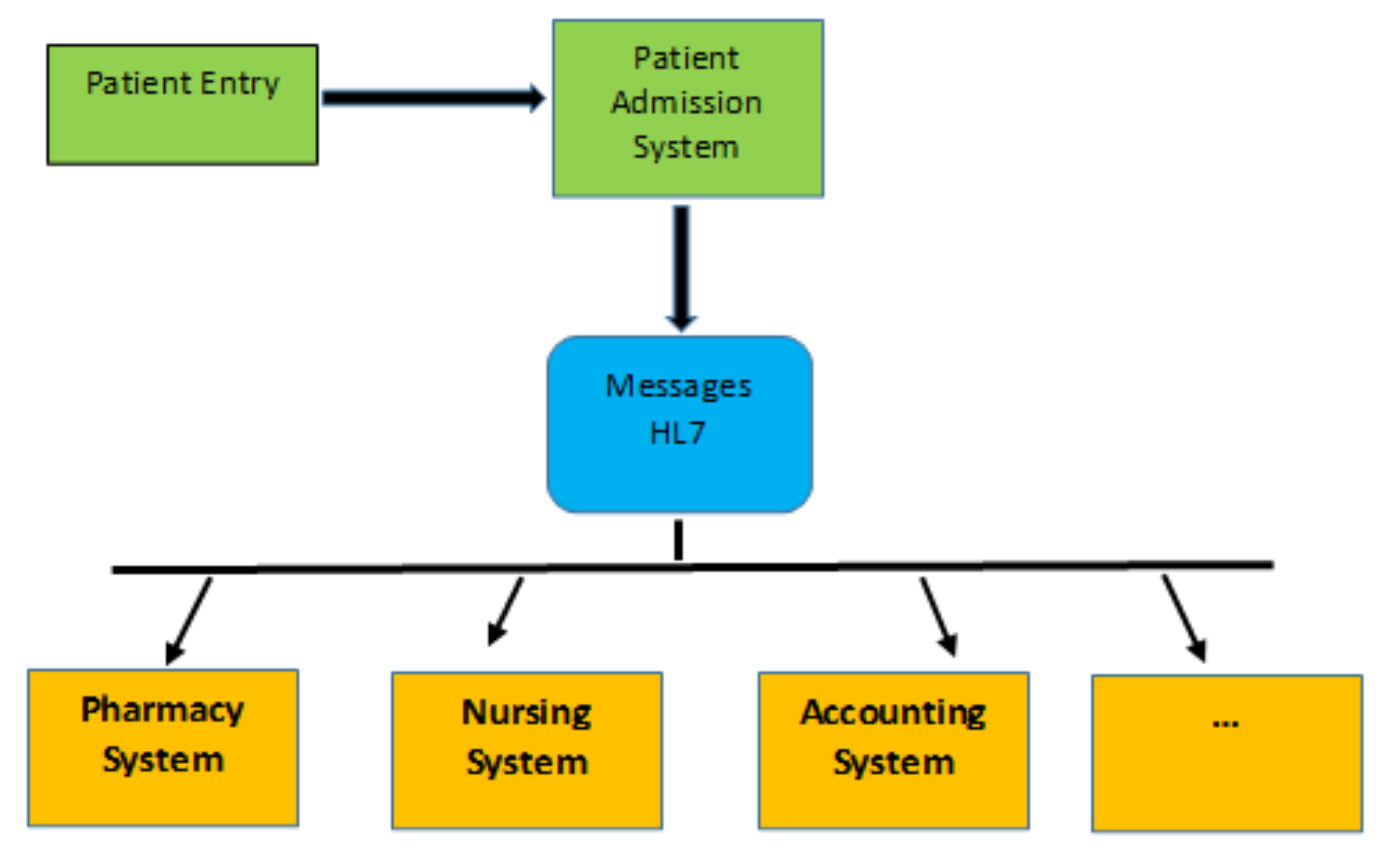

Figure 3: General concepts of the HL7 Source: [HL7 Colombia]

The messages are formed througth data campus of variable longitude that are separated by campus of separator characters. The rules describe how its codify the different types of campus data and when it can be repeted. The data campus formed logical aggrupation called segments, which separate one another through separator characters. Each segment start with a literal value for three longitude characters that identifies the segment on the message. The segments can be repeated and the data campus are localized in one message by its relative position associated by the segments [5]. 


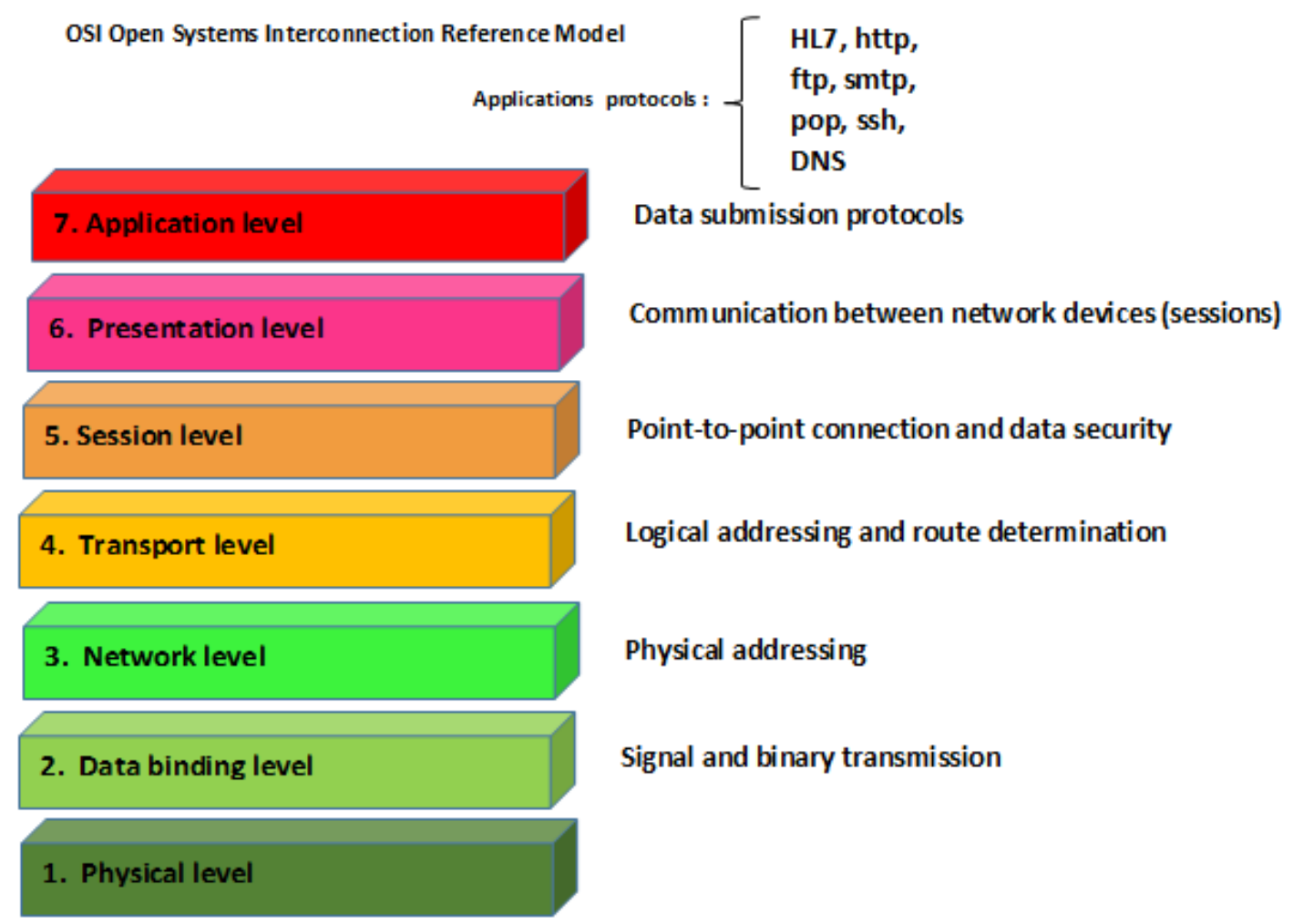

Figure 4: Model of reference for open systems OSI Source: HL7.org

Until now, we have seen the HL7 standard like a model of server-client. However, it can be applied too as a file transference. The messages can be codified on a file and then the file can be transfer by external procedures like can be the FTP, FTAM, etc.

The HL7 standard its written assuming, that and event on the real world (sanity area) created the necessity of a flown of exchange between medical systems. To this event, of the real world we defined it like trigger event.

Interoperability: It's define as the capacity that two or more systems has for the Exchange of information and be used immediately by on its logic of internal process. They are distinguished four types of interoperability: the technic, the semantic, the culture and the organizational. The models are interrelated to offer best and better resolution of the patient problems. On this context, it must adopt standards and models of reference that train the systems and decrease the implementation costs [6].

There is one clinical document of architecture (CDA) which is a generic model for the communication of clinical documents very like the "composition" class on the CEN 13606 specification and the "transaction" class on an open EHR. The original intention was to provide and standardized form to communicate clinical notes, however the community of users of the CDA has tent to use it more like a persistence specification. Some see it as the equivalent HL7 of a historical architecture of clinical electronical history. The edition 2.0 of the CDA defined the structural organization of information of fine-grained inside a document. The work has started with "templates" described as "conditioning models for HL7 specifications" This work it is realized in collaboration with members of the open EHR, and it is starting to include ideas of the methodology of open archetypes EHR [7].

The standard Digital Imaging and Communications or DICOM has been developed for the ACR (American 
College of Radiology) and the NEMA (National Electrical Manufacturers' Association). On its development, it has maintained a high level of collaboration with other organizations of standardization including CEN TC251 in Europa and JIRA in Japan, participating on the revision of other organization of the United Stated as IEEE, HL7 and the ANSI.

DICOM define the communication standards and the message formats for diagnostic and therapeutic messages. DICOM is supported by most manufactures of radiological equipment and the (Picture Archiving and Communications Systems). This standard has been incorporated to the European normative MEDICOM (Medical Image Communication).

The actual standard DICOM version 3.0, collect a big number of improvements related to previous versions that can online be applicable to communication systems. DICOM version 3.0 supports the red work with standards protocols type OSI and TCP/IP. Moreover, the version 3.0 specifies the standard devices that reacts to orders and data through the concept of service class, semantic of the commands and associated data [8].

\section{EXECUTION OF THE RESEARCH WORK}

For the design of message for ambulatory consultation, its necessary to realize a scenery, we describe it then:

Ambulatory consultation message scenario. Everything starts when a patient enters a consulting room, where a doctor attends it, an uprising of information is made, and this information is analyzed by the doctor and documented on the ambulatory consultation document.

For there to be a query, there always must intervene two authors, the doctor and the patient. The doctor after analyzing the information determinates if the patient should send it to a laboratory or to a specialist, this is made through the consultation that was made.

Depending of the entity that provides the health service, there is order to the patient to make the corresponding exams, later they are given to the doctor for him to analyze and the results are deliver on the ambulatory consultation to the patient.

Principal entities and roles on the scenery of ambulatory consultation. The principal entities of the scenery of ambulatory consultation are described then:

Institution providing the health service: It is the institution created to provide the health services to the affiliated and benefactors, on the attention level that corresponds. It must have like principles as the quality and efficiency; it will have administrative, technical and financial autonomy.

Health insurance Company. It is an organization that plays the role of payer of the services of diagnostic proves.

They are classified in:

Entities Promoters of Health: They are administrators of the obligatory plan of social security of health

- EPS of contributive regime.

- EPS of subsidiary regime.

- Insurers of exception regime.

- Insurers of pre-paid medicine. 
Its importance on the laboratory scenery, it is because it requires the payment authorization for providing certain services of diagnostic proves.

Consultant doctor. The consultant doctor is a professional of the health that helps the providers of health to recognized, promote the health, and security of kids and families, and provide a safe place to kids.

The consultant of mental health specialized of the care of kids, Works on the social and emotional health, the wellness, and development. The consultant of mental health helps with problems related to the kids behaved, the child abuse, the stress of the family and the providers of health. It participates as author of this ambulatory consultation.

Specialist. It is called in that way to doctors that are specialist on Medicine, that through an undergraduate program known as hospital resident acquires a set of medical knowledges relative to this specifically area of the human body, some surgical technics and a determinate medical diagnostic.

Laboratory. Called bacteriologist, it is a person that plays the role of entity licensed to realize procedures of laboratory diagnostic. Participates as author of the reports of results, which authentic through its signature, giving them the character of clinical document.

Use case diagram. It was identify principally one actor: The system user, from which they come off the use case (Actual stage of a patient in ambulatory consultation), which interacts with the health center and it's attendance by a doctor, additionally it has been made a detailed description of the use case, from which has created a table that contains the following spaces to fill: CU-XX (Use case number), Description (on this fill its made a short description of what does the use case does), normal sequence (on this fill it's detailed the step by step of the use case), Exceptions (on his fill it is describe the possible failures that the use case can have), performance (it's the time in which is expected that the application make the actions that describes the use case) and for last we found a fill to put the comments that are required to be done about the use case describe. 
Table 1: Description of the using case for a message of ambulatory consultant.

\begin{tabular}{|c|c|c|}
\hline $\mathrm{CU}-001$ & & Scenery \\
\hline Descrip tion & The patient & with the health care center, for being attended by a doctor. \\
\hline \multirow[t]{6}{*}{$\begin{array}{l}\text { Normal } \\
\text { Seq uence }\end{array}$} & Step & Action \\
\hline & 1 & The parient asks for a consult \\
\hline & 2 & $\begin{array}{l}\text { The consult is schedule on the system of the health ca } \\
\text { center. }\end{array}$ \\
\hline & 3 & $\begin{array}{l}\text { The patient is informed with the date, time and place of } t \\
\text { consult. }\end{array}$ \\
\hline & 4 & The partient goes to the consult \\
\hline & 5 & $\begin{array}{l}\text { The doctor realized the procedure of ambulatory consultatic } \\
\text { and gives a result. }\end{array}$ \\
\hline \multirow[t]{2}{*}{ Exceptions } & 1 & The patient that don't attended to the consult. \\
\hline & 2 & Adjoumment of the consult by the health care center- \\
\hline Performance & \multicolumn{2}{|c|}{ The system must do the actions already s aid on the steps one (1) to five (5) } \\
\hline Frequency & \multicolumn{2}{|c|}{ How many time does the patient asks for a conswlt. } \\
\hline Importance & \multicolumn{2}{|c|}{ Guarantee the correct management of the information. } \\
\hline Urgency & \multicolumn{2}{|c|}{ The parient will have to wait or it can be attend ance immediately. } \\
\hline Comments & \multicolumn{2}{|c|}{$\begin{array}{l}\text { The proves will be made when the message of ambulatory consultant gets a } \\
\text { operation. }\end{array}$} \\
\hline
\end{tabular}

Source: Authors

Sequence diagram. The sequence diagram shows the different interactions between the different objects that are part of the message for ambulatory consultation. 


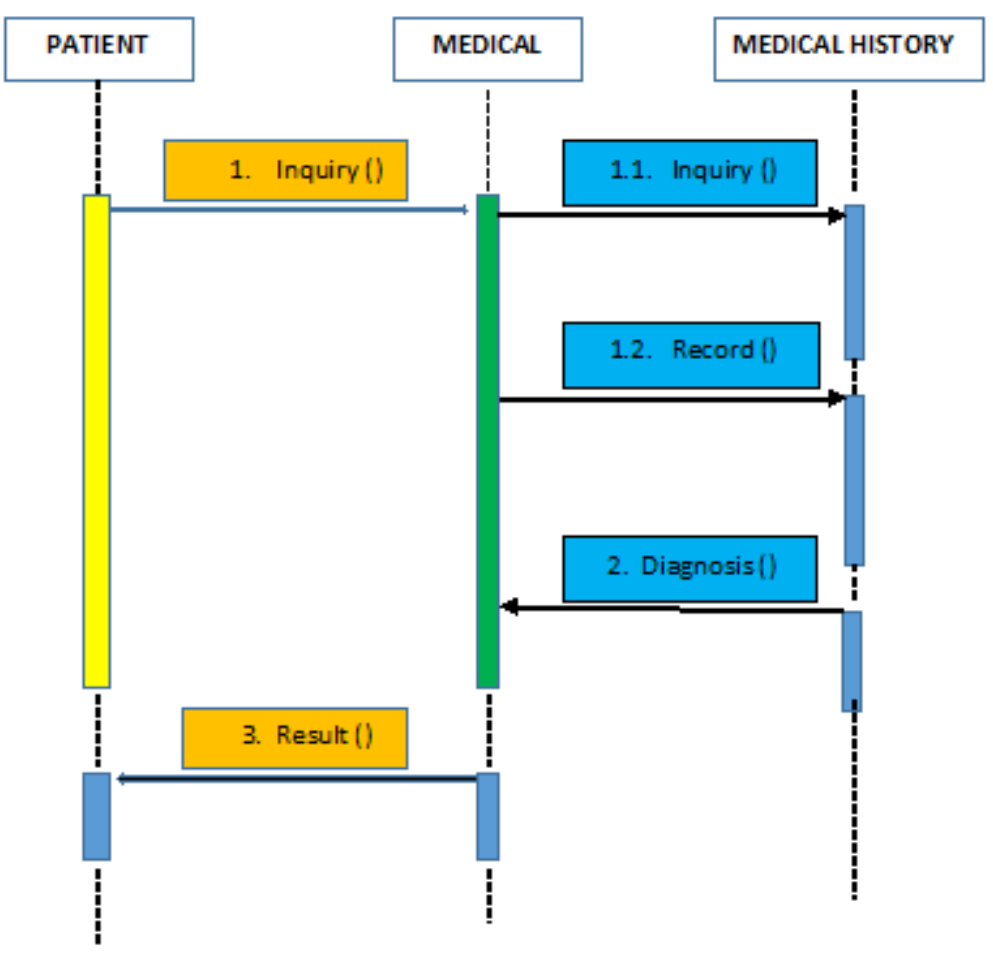

Figure 5: Sequence diagram. Source: Authors, 2011

The range of the message for ambulatory consultation of the standar HL7 v3, counts with a message model and otrher artefacts that are necessary for the support of the message, related with the request of sanitary and asistance services of basical health.

This range include the specification for the Messenger for the request of health care services, including basic services of nursing, therapy, imaging and materials related with the care of the patient.

This are a few information models for the range of messages of specialized orders and observations that has been defined:

- Laboratory.

- $\quad$ Provision of patient attendance (Ambulatory consultation).

- $\quad$ Therapeutically device.

- Blood, Tissues y organs.

- Genomic clinic.

The range for the dominium for the provision of attendance to the patient of the HL7 v3 standard includes the request of various types of health services, among which are the ambulatory consultation. 


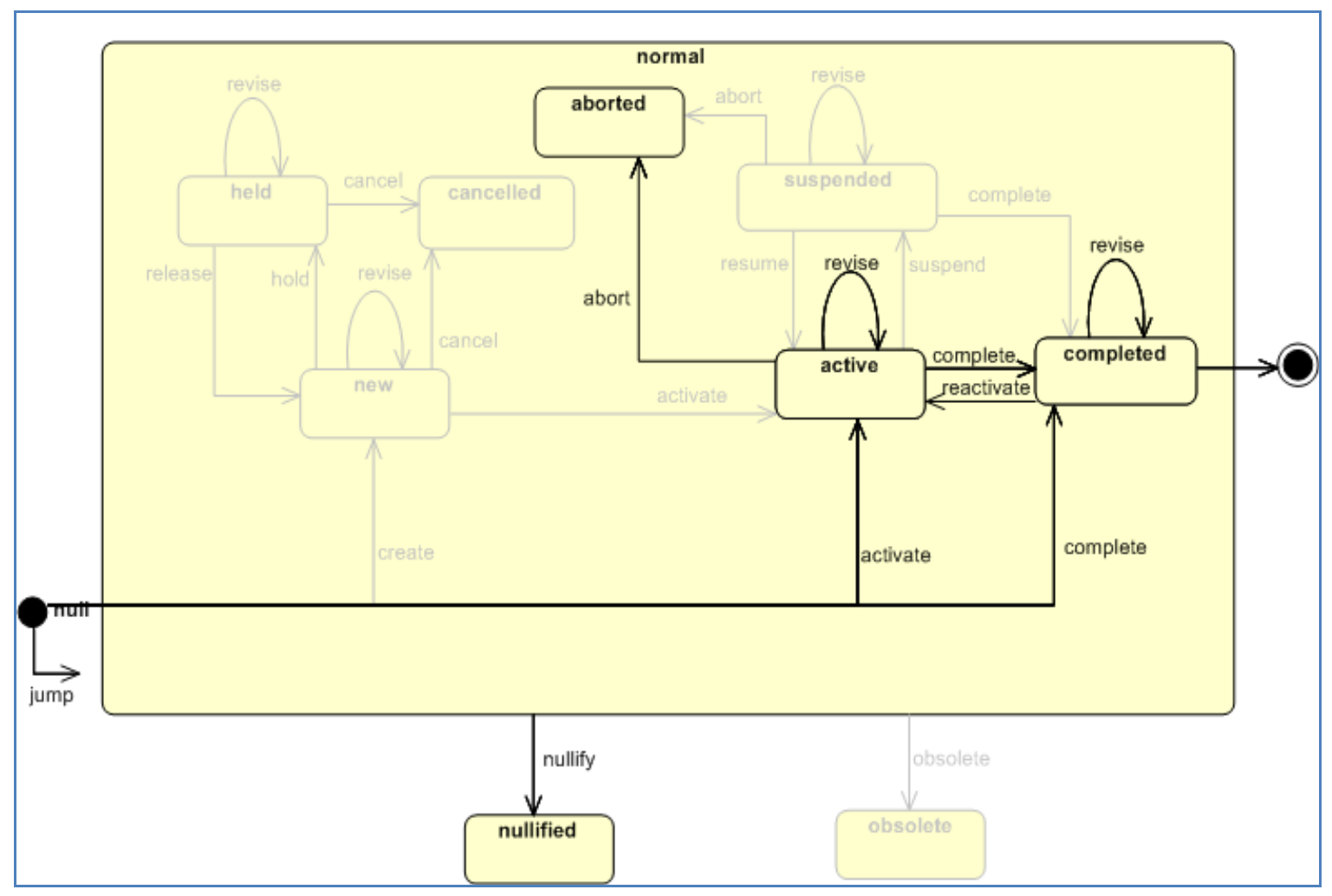

Figure 6: Lifecycle of the message for ambulatory consultant. Source: Hi7.org

Valid the develop message. On this process, it can be verified that it is necessary to establish the process and procedures, as the identification of other messages with which can be interoperation. The proof of the message will be realized on the MARELI platform of the Funtelmed foundation, which have an open system of telemedicine, which is in permanent messages construction; it was made on this point and significant contribution for solving problems of design and implementation of the HL7 messages.

The investigation research and the previous results, gave the methodological and logical contents for constructing a guide that allows the implementation of the message on other systems, and that interacts with the development of the foundation been this and important objective for the HL7, it's implementation.

Finally, the structure of the work allows the development of the work under the systemic focus, which was endorsed for the engineering of the foundation.

On respect of the future works, the results of the research facilitate the continuity of the work, because they supported the development of a standard that is on the start of the implementation and adequacy for Colombia.

\section{CONCLUSIONS}

For designing a prototype of message for ambulatory consultation, they will be developed activities such as the identification of the route of the message on the BALLOT, lifting of requirements, as well as the definition and description of the respective diagrams (Use case, estate case) and it was achieved the construction of the message.

When designing a prototype of message of ambulatory consultation, it ca be verified that it is necessary to stablish the process and procedures, as identifying other messages with which can be interoperation. The prove of the message was 
made on the MARELI platform of the foundation Funtelmed, which has an open system of telemedicine that is on permanent construction of messages, on this sense, a contribution was made into the solution of problems of design and implementation of the HL7 message.

The validation of the message was done, with the support of the personal of the FUNTELMED foundation, which are affiliated to the HL7 in Colombia that was made to verify that the HL7 standard was being fulfilled.

It was carried out the respective documentation to the development of the consultation message, which will make it easy to the medical and computing community of health the interpretation of the message that interoperates on an open way with other systems, because this message gets integrated into a Messenger system for the standardization of all the system and the integration of heterogeneous systems being this message the principal source of the interoperability between this systems on the cases of ambulatory consultation.

The research work, and the previous results gives the methodological and logistic contents to construct a guide that allows the implementation of the message on other systems and that interacts with the development for the foundation being this an important goal for the HL7 and its implementation.

Regarding future Works the results of this investigation, makes it easier to the continuity of the work, insomuch as they support the development of a standard that it is on the start of the implementation and adequacy for Colombia.

The message made for ambulatory consultation comply with the HL7 standard, which has a large universe, therefore, the realization of the message is just one link more in the chain, what it entails to realize the different messages of the diverse sceneries of the Rim dominium, in order to make on a future a standardization of the health systems and to make the possibility to interoperate in the best way of the user service, taking advantage of all the information that flows in the different systems. This will be helpful on the different process needed by the patient.

\section{ACKNOWLEDGMENTS}

The authors would like to thank the Universidad Distrital Francisco José de Caldas and the GITEM research group that supported the development and testing of the project.

\section{REFERENCES}

1. HL7 ARGENTINA. What is the HL7 [Online]. January 1, 2006.

2. http://hl7.org.ar/index.php?option=com_content $\&$ task=view \&id=19\&Itemid $=142$

3. Wordlingo, Level 7 of health [Online]. 〈http://www.worldlingo.com/ma/enwiki/es/Health_Level_7\#Organization>.

4. Ministry of public health standard HL7 [Online]. 〈http://www.di.sld.cu/estandar.php〉.

5. Garcia N. J A., Electronic Exchange of health documents, Level Seven. Article. July 12, 2009

6. Montaged J., Hernandez S. Standard for the electronic clinic history, Health care institute Carlos III, University Hospital Puerta de Hierro. P. 203

7. Ministry of Public Health Standard HL7 [Online]. 〈http://www.di.sld.cu/estandar.php〉.

8. Garcia Naya, José Antonio. Electronic Exchange of health documents, Level Seven. Article. July 12, 2009 
9. Monteagudo José, Hernandez Salvador. Standard for the electronic clinic history, Health care institute Carlos III, University Hospital Puerta de Hierro. P. 203

10. Monteagudo. Op.cit., P. 213 